\title{
EMPIRE ultimate expansion: resonances and covariances
}

\author{
M. Herman ${ }^{1, a}$, S.F. Mughabghab ${ }^{1}$, P. Obložinský ${ }^{1}$, D. Rochman ${ }^{1}$, M.T. Pigni ${ }^{1}$, T. Kawano ${ }^{2}$, R. Capote ${ }^{3}$, V. Zerkin ${ }^{3}$, A. Trkov ${ }^{4}$, \\ M. Sin $^{5}$, B.V. Carlson 6 , H. Wienke ${ }^{7}$, and Young-Sik Cho ${ }^{8}$ \\ 1 National Nuclear Data Center, Brookhaven National Laboratory, Upton, NY, USA \\ 2 T-16, Los Alamos National Laboratory, Los Alamos, NM, USA \\ 3 International Atomic Energy Agency, Nuclear Data Section, Vienna, Austria \\ 4 Jozef Stefan Institute, Ljubljana, Slovenia \\ 5 University of Bucharest, Bucharest, Romania \\ ${ }^{6}$ Instituto Tecnologico de Aeronáutica, São José dos Campos, Brazil \\ 7 Belgonucleaire, 2480 Dessel, Belgium \\ 8 Korea Atomic Energy Research Institute, Nuclear Data Evaluation Lab., Daejeon, Korea
}

\begin{abstract}
The EMPIRE code system is being extended to cover the resolved and unresolved resonance region employing prooven methodology used for the production of new evaluations in the recent Atlas of Neutron Resonances. Another directions of Empire expansion are uncertainties and correlations among them. These include covariances for cross sections as well as for model parameters. In this presentation we concentrate on the Kalman method that has been applied in EMPIRE to the fast neutron range as well as to the resonance region. We also summarize role of the EMPIRE code in the ENDF/B-VII.0 development. Finally, large scale calculations and their impact on nuclear model parameters are discussed along with the exciting perspectives offered by the parallel supercomputing.
\end{abstract}

\section{Introduction}

EMPIRE [1] is a complex system of codes, linked through a series of scripts and graphical interfaces, designed for modeling nuclear reactions. The system is general and flexible, and can be applied to the calculation of neutron capture in the $\mathrm{keV}$ region, as well as for the research of Heavy Ion induced reactions at several hundreds of $\mathrm{MeV}$. Such broad range of incident energies and projectiles is possible thanks to the extensive set of nuclear reaction models that includes all nuclear reaction mechanisms important below $200 \mathrm{MeV}$. Comprehensive library of input parameters and graphic user interface facilitate preparation of the input and operation of the code. The major emphasis is on nuclear data evaluation and large part of the system is dedicated to ENDF-6 formating, checking, plotting, and processing through the NJOY code. In this way, EMPIRE covers the complete evaluation cycle up to the preparation of files for transport calculations.

\section{Recent developments}

Over the recent years, the system has been continuously extended and improved to meet evaluation exigencies posed by the development of ENDF/B-VII.0 [2]. The international team of developers added a number of new features. In particular, use of the DWBA model has been generalized and prompt fission neutron spectra and nu-bars were added as described in another contribution to this conference [8]. Work continued also on microscopic approaches to the fission channel [9]. In the course of the ENDF/B-VII.0 exercise several bugs were identified and fixed. The code became more reliable and robust

\footnotetext{
${ }^{a}$ Presenting author, e-mail: mwherman@bnl.gov
}

Below, we give a very brief summary of selected changes and elaborate more on ENDF/B-VII.0 and two new fields of expansion: neutron resonances and cross section covariances.

- Automatic fitting of optical potential parameters has been introduced in addition to the earlier manual version. The fitting is highly automatized - user must only select parameters to be varied and EMPIRE extracts useful experimental data from the EXFOR library, sets suitable incident energy grid and performs minimization. Fitting is performed using Coupled-Channels (CC) model with the same coupling scheme, the same models and the same parameters as those used in the actual calculations. The procedure is stable (conservative) i.e., if no clear improvement is found EMPIRE retains initial parameters.

- Full support for dispersive CC optical potentials has been introduced. This modification allows different geometry for the real and the imaginary volume potentials. It enables EMPIRE to utilize physically sound class of optical model potentials that proved to give excellent results with fewer parameters.

- Deformed Multistep Direct model is an extension of the original TUL approach to account for the effect of nuclear deformation. The RPA response functions are calculated in the space of single-particle levels and wave functions derived from the deformed Hamiltonian. The modified model successfully reproduced spectra of inelastically scattered neutrons on ${ }^{232} \mathrm{Th}[6]$.

- Improvements in ENDF-6 formatting and plotting were introduced while developing the ENDF/B-VII.0 library. Here we mention only a mixed representation for storing spectra in the ENDF-6 format. This procedure separates reaction channels into exclusive and inclusive and stores the respective spectra in one of the two formats throughout 
the whole energy range. This approach avoids artificial change of the representation at $20 \mathrm{MeV}$ typically encountered in ENDF evaluations extending to higher energies.

\section{ENDF/B-VII.0 evaluations}

Development of the ENDF/B-VII.0 was an occasion for a first large scale deployment of the EMPIRE code. It has been used for 77 out of 393 neutron evaluations contained in the library. These new evaluations were produced by the following laboratories:

- 25 materials were evaluated by BNL,

- 37 materials were evaluated by BNL in collaboration with KAERI, S. Korea (32 materials) [4] and with JAERI renamed JAEA, Japan (5 materials),

- 8 materials were evaluated by BNL, including covariances produced by BNL-ORNL-LANL collaboration, and

- 4 materials of specific interest were evaluated by LLNL $\left({ }^{74,75} \mathrm{As}\right), \mathrm{LANL}-\mathrm{BNL}\left({ }^{89} \mathrm{Y}\right)$ and BNL $\left({ }^{90} \mathrm{Zr}\right)$,

- 3 actinides $\left({ }^{232} \mathrm{Th}\right.$ and $\left.{ }^{231,233} \mathrm{Pr}\right)$ including covariances were evaluated at IAEA.

In most cases these are completely new files up to $20 \mathrm{MeV}$, in which fast neutron range and sometimes also unresolved resonance range are fully based on the EMPIRE calculations. The basic role of the experimental data was to determine model parameters. In some cases minor adjustments were imposed on the calculated results in order to match high quality experimental data. Such adjustments were carried out during the calculations so that they were consistently propagated onto all reaction channels.

The EMPIRE based evaluations are complete: they include all reaction channels of importance for neutronics calculations, angular distributions, double-differential spectra, and recoils. The photon production spectra are provided for all reaction channels involving emission of $\gamma$-rays. The results were obtained using up to date reaction theory including CC and DWBA models in the incident channel and inelastic scattering, quantum-mechanical preequilibrium emission of neutrons (Multistep Direct and Multistep Compound), exciton model for emission of protons, $\alpha$-particles (the latter with Iwamoto-Harada extension) and photons, widths fluctuation correction at incident energies below $3 \mathrm{MeV}$ and sequential emission Hauser-Feshbach with full $\gamma$-cascade. For the materials of high priority the optical model parameters were adjusted in the course of evaluation. In general, we preferred to perform evaluation for the whole chain of isotopes since this allows for a more reliable constrain of model parameters and ensures consistency between the isotopes. In figure 1 we show, as an example, capture cross sections for the extensive set of gadolinium isotopes.

\section{Resonance module}

EMPIRE-2.19 has been using an internal, ENDF-6 formatted, database of resonances to complete fast neutron evaluations with the resonance region. Recently, Mughabghab completed

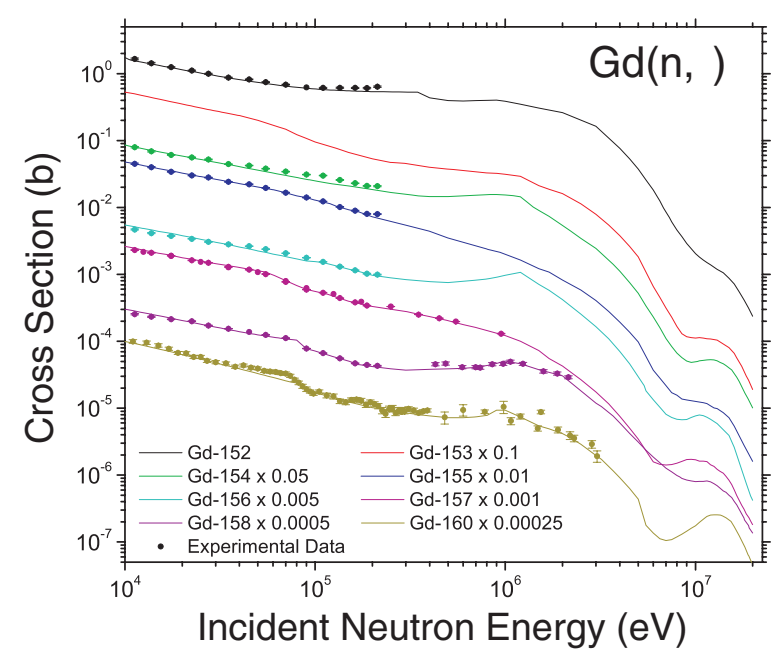

Fig. 1. Capture cross sections for Gd isotopes compared to experimental data in the fast neutron region. The neighboring curves and data are scaled by factors indicated in the legend.

his Atlas of Neutron Resonances [3], a monumental compilation/evaluation work across the whole table of isotopes. This wealth of information must find its way into the evaluated libraries. With this goal in mind, we have started a new EMPIRE module [7] that will provide easy access to the Atlas data and their seemless transfer to the evaluation. We also intend to preserve legacy codes and methodology developed over years and constituting scientific heritage of great importance for application of nuclear technology. We stress that the new module is intended for extracting and further processing of resonance parameters and properties collected in Atlas, i.e., it is not replacing codes used to analyze experimental data such as SAMMY or REFIT.

Contents of the Atlas includes actual resonance parameters (energies and widths along with uncertainties) and resonance properties (thermal cross sections, scattering radius, average resonance spacings, and average radiative, neutron and fission widths for s-, p-, and d-waves). The task of extracting, analyzing and formating these quantities is delegated to the codes PTANAL and WRIURR that run in sequence supported by a few utility codes.

PTANAL assigns orbital momentum and spins to resonances if such were not determined from measurements. When doing it PTANAL makes use of the Bayesian method and random assignment, respectively. In addition, average radiation width is calculated and assigned to the resonances with unknown radiative width and the fitting of reduced neutron widths to the Porter-Thomas distribution is performed. The completed set of resonances is stored in the ENDF-6 format (File 2) and passed to the WRIURR code along with the average resonance parameters. WRIURR constructs the unresolved resonance region using average parameters and appends it to the ENDF file.

RECENT, LINEAR and SIGMA1 codes are used to reconstruct pointwise cross sections for plotting against experimental data retrieved automatically from the EXFOR library. Graphic user interface (GUI) will allow the evaluator to adjust boundaries of resolved and unresolved resonance regions, the average resonance parameters and their energy dependencies 


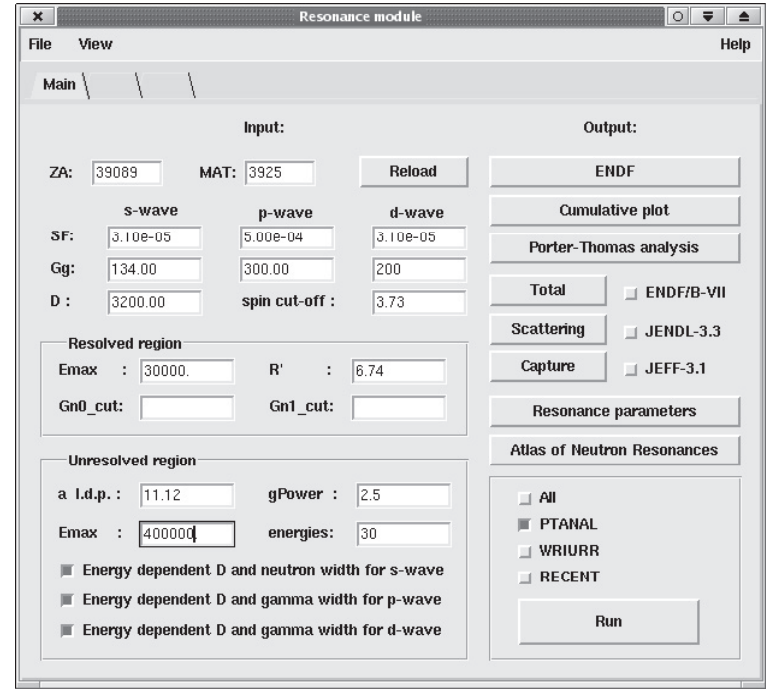

Fig. 2. Conceptual design of the future graphic user interface controlling the resonance module of EMPIRE. Average resonance parameters are retrieved from the Atlas and can be adjusted by the evaluator. Statistical analysis of the resolved resonances (cumulative plots and Porter-Thomas analysis) will be accessible through a click of a button together with the graphical comparison of reconstructed cross sections with experimental data and other evaluations.

in order to reproduce measured cross sections. The concept GUI for the resonance module is shown in figure 2. Merging of the resonance region with the fast-neutron part of the evaluation will be performed with the existing code ENDRES.

\section{Covariance module}

The methodology for generating cross section covariances in the fast neutron range was developed recently by the National Nuclear Data Center (BNL) in collaboration with the T-16 group (LANL) [5]. A sensitivity matrix is produced with the code EMPIRE, and used in the KALMAN code for determining covariances while taking into account relevant experimental data.

To obtain the sensitivity matrix, the most relevant model parameters in EMPIRE (optical model, level density and preequilibrium strength) are varied independently around the optimal value, to determine their effect on cross sections (see figs. 3 and 4). Sensitivity matrix elements are calculated as a change of a given reaction cross sections in response to the change of a particular model parameter. A series of scripts is employed to transfer such sensitivity matrix information along with the experimental data to the KALMAN code.

Figure 5 shows an example of the correlation matrix obtained by combining model calculations with experiment. We note, that final uncertainties are often adjusted to reproduce error bars on the best measurements by preventing errors on model parameters (initially set at 10\%) from falling below reasonable limits (3\%). This procedure is invoked since the Kalman filter tends to reduce uncertainties on model parameters if many consistent experimental data are well reproduced by the model calculations.

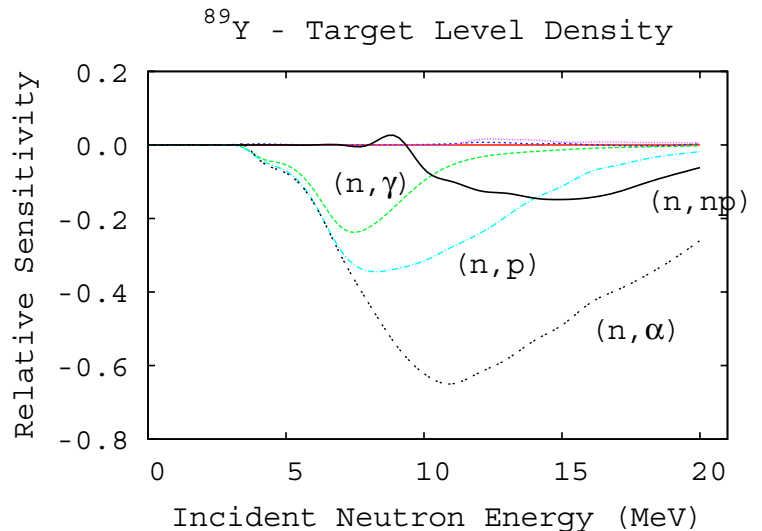

Fig. 3. Relative sensitivity of various reaction channels to the perturbation of the level density parameter in the target nucleus $\left({ }^{89} \mathrm{Y}\right)$ by $10 \%$.

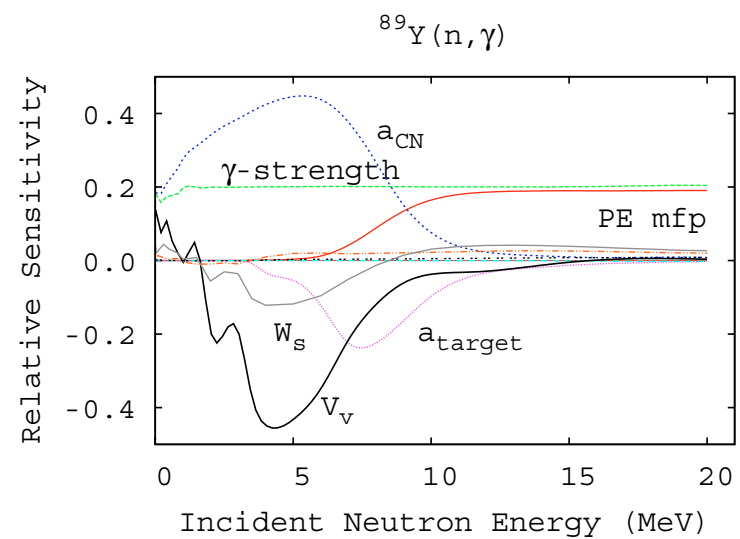

Fig. 4. Relative sensitivity of neutron capture on ${ }^{89} \mathrm{Y}$ to the $10 \%$ perturbation of various model parameters $(\gamma$-strength perturbed by $20 \%$ ). Below $10 \mathrm{MeV}$ (the compound nucleus regime) capture is determined mostly by level density parameter in the compound nucleus $\left(a_{C N}\right)$ and depth of the real optical model potential for neutrons $\left(V_{v}\right)$ with target level density and depth of the imaginary surface potential plying lesser role. Above $10 \mathrm{MeV}$ capture is dominated by the preequilibrium mechanism and appears to be mostly sensitive to the mean free path (PE mfp). $\gamma$-ray strength function contribution is constant over nearly entire energy range.

The sensitivity matrices turn out to be a very valuable off-spin of the covariance calculations. They allow for a deeper insight into the role of different reaction mechanisms, and offer extremely useful guidance for adjusting model parameters. Full set of sensitivity plots allows to determine which parameters should be changed in order to achieve required change in the cross section. By the same token, the undesired changes in other reaction channels can be predicted and avoided. Figures 3 and 4 show that the effect of variation of some of the parameters reveal marked and difficult to anticipate energy dependence. Therefore, combined adjustment of several parameters might be very effective in reproducing experimental data with theory. In addition, simultaneous fitting of various reaction channels is likely to reduce ambiguity of such a fit. We note that essential function of the Kalman 


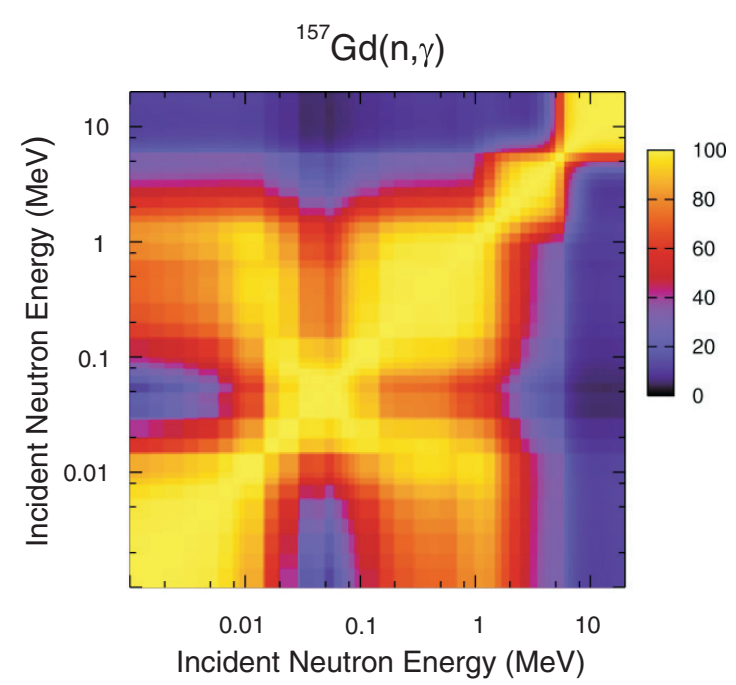

Fig. 5. Correlation matrix for the ${ }^{157} \mathrm{Gd}$ neutron capture cross sections in the fast neutron region obtained with the EMPIRE-KALMAN method.

filter is to perform adjustment of the parameters to match all available data as close as possible. Therefore, Kalman output contains optimal values of the parameters along with their respective covariances in addition to covariances for the cross sections. If such an adjustment is done on a large scale it should produce improved global set of model parameters.

\section{Ultimate vision}

Modern evaluations in the fast neutron region tend to rely on nuclear reaction theory with experimental data being critical for the calibration of model parameters. Then the library is validated against integral measurements, generally much more precise than the microscopic ones. However, integral measurements integrate over many nuclides, reactions and incident energies that makes tracing eventual discrepancies back to the microscopic data generally very difficult and in most cases inconclusive.

An alternative approach is to incorporate integral measurements into the model assisted evaluation procedure in order to achieve such a precision of the microscopic data that there is no need for any adjustments to reproduce integral experiments. The essential advantage of such a procedure would be adjusting of a few model parameters rather than continues cross sections. Recent progress in nuclear reaction modeling, qualitative jump in the computation resources, status of modern transport simulation codes, and availability of key libraries such as RIPL (model parameters), EXFOR (microscopic experimental data) as well as the extensive compilation of benchmark experiments (ICSBEP) provide justified expectation that such an ambition task is technically feasible in the fast neutron range. Our confidence builds on the follwing:

- simultaneous evaluation (model calculations) of the entire library (or a considerable part of it) and its benchmarking against hundreds of integral measurements will considerably increase constrains on the adjustments of the microscopic data,

- adjustment of model parameters instead of cross sections will greatly reduce number of quantities to be adjusted reinforcing the effect of the previous point,

- comprehensive sensitivity calculations coupled to the advanced statistical methods (e.g., KALMAN filtering) will ensure that the adjustment is optimal.

Such a project, known as Global Nuclear Data Initiative, has recently been gaining support within the US nuclear data community.

\section{Conclusions}

The EMPIRE code has reached sufficient level of robustness and reliability to provide substantial support for the development of ENDF/B-VII.0. Several important extensions and improvements were introduced. Recently, the code has been embracing new territories: neutron resonances and the covariances. Our vision for future goes even further - consider the library as a single entity, rather than as the collection of isotopes. The dream is to construct consistent set of input files (including resonance parameters) that, together with the nuclear reaction code, could replace the entire library of cross sections. Ultimately, if the physics is right, there should only be one input in the fast neutron region!

\section{References}

1. M. Herman, R. Capote, B. Carlson, P. Obložinský, M. Sin, A. Trkov, V. Zerkin, EMPIRE nuclear reaction model code, version 2.19 (Lodi), www.nndc.bnl.gov/empire219/ (2005).

2. M. Chadwick, P. Obložinský, M. Herman et al., Nucl. Data Sheets 107(12), 2931 (2006).

3. S.F. Mughabghab, Atlas of Neutron Resonances: Thermal Cross Sections and Resonance Parameters (Elsevier, Amsterdam, 2006).

4. H.I. Kim et al. (these proceedings).

5. D. Rochman et al. (these proceedings).

6. H. Wienke, R. Capote, M. Herman, M. Sin (these proceedings).

7. Young-Sik Cho, M. Herman, S.F. Mughabghab, P. Obložinský, D. Rochman, Y.O. Lee (these proceedings).

8. R. Capote, Mihaela Sin, A. Trkov, M. Herman, B.V. Carlson, P. Obložinský (these proceedings).

9. R. Capote, S. Goriely, S. Hilaire, A. Koning, M. Sin (these proceedings). 